\title{
Strong convergence theorem for common solutions to quasi variational inclusion and fixed point problems
}

\author{
Xianzhi Tanga, Huanhuan Cuib,* \\ ${ }^{a}$ Department of of basic courses, Yellow River Conservancy Technical Institute, Kaifeng 475004, China. \\ ${ }^{b}$ Department of Mathematics, Luoyang Normal University, Luoyang, 471022, China.
}

Communicated by Y. H. Yao

\begin{abstract}
In this paper, we consider a problem that consists of finding a common solution to quasi variational inclusion and fixed point problems. We first present a simple proof to the strong convergence theorem established by Zhang et al. recently. Next, we propose a new algorithm to solve such a problem. Under some mild conditions, we establish the strong convergence of iterative sequence of the proposed algorithm. (C)2016 all rights reserved.
\end{abstract}

Keywords: Variational inclusion, fixed point problem, inverse strongly monotone operator, nonexpansive mapping, multi-valued maximal monotone mapping.

2010 MSC: 47H05, 47H09, 47J05, 49J25.

\section{Introduction}

In this paper, we consider a quasi variational inequality problem that requires to find a point $u \in \mathcal{H}$ so that

$$
\theta \in A(u)+M(u),
$$

where $\mathcal{H}$ is a real Hilbert space, $A: \mathcal{H} \rightarrow \mathcal{H}$ is a single-valued mapping and $M: \mathcal{H} \rightarrow 2^{\mathcal{H}}$ is a multi-valued mapping. The solution set of problem (1.1) is denoted by $V I(\mathcal{H}, A, M)$. We recall one of special cases of

\footnotetext{
${ }^{*}$ Corresponding author

Email address: hhcui@live.cn (Huanhuan Cui)
} 
such problem. If $M=\partial \delta_{C}$ with $C$ a nonempty closed convex subset of $\mathcal{H}$, and $\delta_{C}: \mathcal{H} \rightarrow[0,+\infty]$ is the indicator function of $C$, that is,

$$
\delta_{C}(x)= \begin{cases}0, & x \in C, \\ +\infty, & x \notin C .\end{cases}
$$

In this case, problem 1.1 is reduced to finding a point $u$ so that

$$
\langle A(u), v-u\rangle \geq 0, \forall v \in C,
$$

which is called Hartman-Stampacchia variational inequality problem. A fixed point problem requires to find a point $u$ so that

$$
S u=u,
$$

where $S: \mathcal{H} \rightarrow \mathcal{H}$ is a nonlinear mapping. The set of fixed points of $S$ is denoted by $F(S)$.

Takahashi and Toyoda [6] considered the problem for finding a common solution to Hartman-Stampacchia variational inequality problem $(1.2)$ and fixed point problem $(1.3)$, that is, find a point $u$ such that

$$
u \in F(S) \text { and }\langle A(u), v-u\rangle \geq 0, \forall v \in C .
$$

Since then much efforts have gone into constructing algorithms to solve such a problem; see e.g., [3, 5, 6, 10] and references therein. Recently, Zhang et al. [11] considered a problem to find a common solution of problems (1.1) and (1.3), that is, find a point $u$ such that

$$
u \in F(S) \cap V I(\mathcal{H}, A, M) .
$$

It is obvious that problem (1.5) is an extension of the problem (1.4 considered by Takahashi and Toyoda 6]. In [11], Zhang et al. constructed an algorithm, which generates a sequence $\left(x_{n}\right)$ by

$$
\left\{\begin{array}{l}
x_{0}=x \in \mathcal{H}, \\
y_{n}=J_{M, \lambda}\left(x_{n}-\lambda A x_{n}\right), \\
x_{n+1}=\alpha_{n} x+\left(1-\alpha_{n}\right) S y_{n},
\end{array}\right.
$$

where $J_{M, \lambda}$ is the resolvent related to $M$ with $\lambda$ a positive constant. Under some certain assumptions, they proved the sequence $\left(x_{n}\right)$ generated by (1.6) converges in norm to a solution of problem (1.5).

The aim of this paper is to introduce some new iterative algorithms to solve problem (1.5). We first prove the strong convergence of algorithm $(1.6)$ by employing a new simple proof. Some other iterative schemes to approximate the solution of problem (1.5) are proposed and also the strong convergence properties for these new algorithms are proved.

\section{Preliminaries}

Throughout this paper, $I$ denotes the identity operator on $\mathcal{H}$, " $\rightarrow$ " strong convergence, " $\rightarrow$ " weak convergence, and $\omega_{w}\left(x_{n}\right)$ the set of weak cluster points of the sequence $\left(x_{n}\right)$. Let $P_{C}$ denote the projection from $\mathcal{H}$ onto a nonempty closed convex subset $C$ of $\mathcal{H}$, that is,

$$
P_{C} x=\underset{y \in C}{\arg \min }\|x-y\|, \quad x \in \mathcal{H} .
$$

It is well-known that $P_{C} x$ is characterized by the inequality:

$$
\left\langle x-P_{C} x, c-P_{C} x\right\rangle \leq 0, \quad c \in C .
$$

Let $T$ be a mapping defined on $\mathcal{H}$. Recall that $T$ is contractive if there is a $\kappa \in(0,1)$ so that $\|T x-T y\| \leq$ $\kappa\|x-y\|$ for any $x, y \in \mathcal{H}$; and nonexpansive if $\|T x-T y\| \leq\|x-y\|$ for any $x, y \in \mathcal{H}$. The fixed point 
problem (1.3) for the nonexpansive mapping has been widely investigated and studied. There are two iterative schemes with strong convergence for approximating a fixed point of a nonexpansive mapping. One is the Halpern iteration, which generates an iterative sequence by

$$
x_{n+1}=\alpha_{n} x+\left(1-\alpha_{n}\right) S x_{n}, x \in \mathcal{H} .
$$

This iteration is originally constructed by Halpern [2] and further studied by Wittmann [7] and Xu [8]. It is well-known that if $F(S) \neq \emptyset$, then the sequence $\left(x_{n}\right)$ generated by 2.2 converges strongly to $P_{F(S)} x$, whenever $\left(\alpha_{n}\right)$ is a sequence in $(0,1)$ satisfying the following conditions:

(C1) $\lim _{n \rightarrow \infty} \alpha_{n}=0, \sum_{n=0}^{\infty} \alpha_{n}=\infty$;

(C2) either $\sum_{n=0}^{\infty}\left|\alpha_{n+1}-\alpha_{n}\right|<\infty$ or $\lim _{n \rightarrow \infty}\left|\alpha_{n+1}-\alpha_{n}\right| / \alpha_{n}=0$.

Another is viscosity approximation method, which generates an iterative sequence by

$$
x_{n+1}=\alpha_{n} f\left(x_{n}\right)+\left(1-\alpha_{n}\right) S x_{n}, x \in \mathcal{H},
$$

where $f$ is a contraction. This iteration is originally proposed by Moudafi [4] and further studied by Xu [9]. It is well-known that if $F(S) \neq \emptyset$, then the sequence $\left(x_{n}\right)$ generated by 2.3$)$ converges in norm to $P_{F(S)} f$.

A mapping $T$ is called $\nu$-averaged if there exist a constant $\nu \in(0,1)$ and a nonexpansive mapping $S$ such that $T=(1-\nu) I+\nu S$; and $\nu$-inverse strongly monotone $(\nu$-ism) if there is a constant $\nu>0$ such that $\langle T x-T y, x-y\rangle \geq \nu\|T x-T y\|^{2}$ for any $x, y \in \mathcal{H}$. The following lemma collects some useful properties of averaged and inverse-strongly mappings.

Lemma 2.1 ([1]). The following assertions hold.

(i) $T$ is averaged if and only if $I-T$ is $\nu$-ism for some $\nu>1 / 2$;

(ii) The composition of two averaged mappings is also averaged;

(iii) If $T$ is $\nu$-ism with $\nu>0$ and if $\lambda>0$, then $\lambda T$ is $(\nu / \lambda)$-ism;

(iv) If $T$ is 1-ism with $\nu>0$, then it is averaged;

(v) If $T$ is $\nu$-averaged with $\nu \in(0,1)$, there holds the inequality:

$$
\|T x-z\|^{2} \leq\|x-z\|^{2}-\frac{1-\nu}{\nu}\|T x-x\|^{2},
$$

where $x \in \mathcal{H}$ and $z \in F(T)$.

Let $M: \mathcal{H} \rightarrow 2^{\mathcal{H}}$ be a multi-valued maximal monotone mapping. Then the mapping $J_{M, \lambda}$ defined by

$$
J_{M, \lambda}(u)=(I+\lambda M)^{-1}(u), u \in \mathcal{H},
$$

is called the resolvent operator associated with $M$, where $\lambda$ is any given positive constant. The mapping $J_{M, \lambda}$ has the following properties:

Lemma 2.2. Let $A$ be $\alpha$-ism and let $\lambda \in(0,2 \alpha)$. Then the following assertions hold.

(i) $J_{M, \lambda}$ is single-valued and 1-ism;

(ii) $V I(\mathcal{H}, A, M)=F\left(J_{M, \lambda}(I-\lambda A)\right)$;

(iii) $J_{M, \lambda}(I-\lambda A)$ is averaged.

Proof. Assertions (i) and (ii) are proved in [11. Since $A$ is $\alpha$-ism, $\lambda A$ is $\alpha / \lambda$-ism (Lemma 2.1(iii)). It is easy to check that $\alpha / \lambda>1 / 2$, and hence by Lemma 2.1 (i), $I-\lambda A$ is averaged. Since $J_{M, \lambda}$ is 1 -ism, then it is also averaged (Lemma 2.1 (iv)) and therefore by Lemma 2.1 (ii), the composition $J_{M, \lambda}(I-\lambda A$ ) is averaged, too.

The following lemmas will be used in the subsequent section. 
Lemma 2.3 (demiclosedness principle). Let $T: \mathcal{H} \rightarrow \mathcal{H}$ be a nonexpansive mapping with $F(T) \neq \emptyset$. If $\left(x_{n}\right)$ is a sequence in $\mathcal{H}$ so that $x_{n} \rightarrow x$ and $(I-T) x_{n} \rightarrow 0$, then $x \in F(T)$.

Lemma $2.4([8])$. Let $\left(a_{n}\right)$ be a nonnegative real sequence satisfying

$$
a_{n+1} \leq\left(1-\alpha_{n}\right) a_{n}+\alpha_{n} \mu_{n},
$$

where the sequences $\left(\alpha_{n}\right) \subset(0,1)$ and $\left(\mu_{n}\right)$ satisfy the conditions:

(i) $\sum_{n=0}^{\infty} \alpha_{n}=\infty, \lim _{n \rightarrow \infty} \alpha_{n}=0$;

(ii) either $\sum_{n=0}^{\infty}\left|\alpha_{n} \mu_{n}\right|<\infty$ or $\varlimsup_{n \rightarrow \infty} \mu_{n} \leq 0$.

Then $\lim _{n \rightarrow \infty} a_{n}=0$.

\section{Main results}

In this section we first give a simple proof of [11, Theorem 2.1]. The key of the proof is the following lemma.

Lemma 3.1. Let $A$ be a nonexpansive operator and $B$ a $\nu$-averaged operator. If $F(A) \cap F(B) \neq \emptyset$, then $F(A) \cap F(B)=F(A B)$.

Proof. It is obvious that $F(A) \cap F(B) \subseteq F(A B)$. To see the converse, let $x \in F(A B)$. Since $F(A) \cap F(B) \neq \emptyset$, we can pick $u \in F(A) \cap F(B)$. Hence

$$
\begin{aligned}
\|x-u\|^{2}+\frac{1-\nu}{\nu}\|B x-x\|^{2} & =\|A(B x)-u\|^{2}+\frac{1-\nu}{\nu}\|B x-x\|^{2} \\
& =\|A(B x)-A u\|^{2}+\frac{1-\nu}{\nu}\|B x-x\|^{2} \\
& \leq\|B x-u\|^{2}+\frac{1-\nu}{\nu}\|B x-x\|^{2} \\
& \leq\|x-u\|^{2},
\end{aligned}
$$

where the last inequality follows from Lemma 2.1 (v). This implies that $\|B x-x\| \leq 0$, or equivalently, $B x=x$ and further

$$
x=A(B x)=A x .
$$

Altogether we get the result as desired.

Remark 3.2. In [1], Byrne proved that if $\mathrm{A}$ and $B$ are averaged and if $F(A) \cap F(B) \neq \emptyset$, then the intersection $F(A) \cap F(B)$ and $F(A B)$ are coincident. So our result is an extension of this assertion.

Theorem 3.3. Let $A: \mathcal{H} \rightarrow \mathcal{H}$ be $\alpha$-ism with $\alpha>0, M: \mathcal{H} \rightarrow 2^{\mathcal{H}}$ a maximal monotone mapping, and $S: \mathcal{H} \rightarrow \mathcal{H}$ a nonexpansive mapping. If $\left(\alpha_{n}\right)$ is chosen in $(0,1)$ so that the conditions $(\mathrm{C} 1)$ and $(\mathrm{C} 2)$ are satisfied, then the sequence $\left(x_{n}\right)$ generated by 1.6 converges strongly to $x^{*}=P_{F(S) \cap V I(\mathcal{H}, A, M)} x$, whenever $F(S) \cap V I(\mathcal{H}, A, M) \neq \emptyset$.

Proof. Set $T=S J_{M, \lambda}(I-\lambda A)$. Since $S$ and $J_{M, \lambda}(I-\lambda A)$ are both nonexpansive, the operator $T$ is nonexpansive, too. Thus algorithm (1.6) has the following form:

$$
x_{n+1}=\alpha_{n} x+\left(1-\alpha_{n}\right) T x_{n},
$$

which is a standard iterative scheme of Halpern iteration. Since $J_{M, \lambda}(I-\lambda A)$ is averaged, it follows from Lemmas 3.1 and 2.2 that

$$
F(T)=F(S) \cap V I(\mathcal{H}, A, M) \neq \emptyset .
$$

The sequence $\left(x_{n}\right)$ therefore converges in norm to $P_{F(S) \cap V I(\mathcal{H}, A, M)} x$. 
Remark 3.4. Here we choose $\lambda \in(0,2 \alpha)$, while it is assumed that $\lambda \in(0,2 \alpha]$ in [11]. We show that $\lambda$ can not be equal to $2 \alpha$. In fact, it is proved in [11, page 577 , line 11]

$$
\left(1-\alpha_{n}\right) \lambda(2 \alpha-\lambda)\left\|A x_{n}-A u\right\|^{2} \rightarrow 0, \text { as } n \rightarrow \infty,
$$

from which they obtained $\left\|A x_{n}-A u\right\| \rightarrow 0$ as $n \rightarrow \infty$. So, if $\lambda=2 \alpha$, one can not deduce $\left\|A x_{n}-A u\right\| \rightarrow 0$ as $n \rightarrow \infty$.

Theorem 3.5. Let $A: \mathcal{H} \rightarrow \mathcal{H}$ be $\alpha$-ism with $\alpha>0, M: \mathcal{H} \rightarrow 2^{\mathcal{H}}$ a maximal monotone mapping and $S: \mathcal{H} \rightarrow \mathcal{H}$ a nonexpansive mapping. Choose $\lambda \in(0,2 \alpha)$ and define a sequence $\left(x_{n}\right)$ by the iterative procedure:

$$
\left\{\begin{array}{l}
x_{0}=x \in \mathcal{H} \\
y_{n}=J_{M, \lambda}\left(x_{n}-\lambda A x_{n}\right) \\
x_{n+1}=S\left(\alpha_{n} x+\left(1-\alpha_{n}\right) y_{n}\right) .
\end{array}\right.
$$

If $\left(\alpha_{n}\right)$ is chosen in $(0,1)$ so that the conditions $(\mathrm{C} 1)$ and $(\mathrm{C} 2)$ are satisfied, then the sequence $\left(x_{n}\right)$ generated by (3.1) converges strongly to $x^{*}=P_{F(S) \cap V I(\mathcal{H}, A, M)} x$, whenever $F(S) \cap V I(\mathcal{H}, A, M) \neq \emptyset$.

Proof. Take $u \in F(S) \cap V I(\mathcal{H}, A, M)$. We divide our proof into several steps.

SteP 1. The sequence $\left(x_{n}\right)$ is bounded.

Since $J_{M, \lambda}(I-\lambda A)$ is nonexpansive, we have

$$
\left\|y_{n}-u\right\|=\left\|J_{M, \lambda}(I-\lambda A) x_{n}-u\right\| \leq\|x-u\|,
$$

which implies that

$$
\begin{aligned}
\left\|x_{n+1}-u\right\| & \leq\left\|\alpha_{n} x+\left(1-\alpha_{n}\right) y_{n}-u\right\| \\
& \leq \alpha_{n}\|x-u\|+\left(1-\alpha_{n}\right)\left\|x_{n}-u\right\| \\
& \leq \max \left\{\|x-u\|,\left\|x_{n}-u\right\|\right\} \\
& \vdots \\
& \leq \max \left\{\left\|x_{0}-u\right\|,\|x-u\|\right\}=\|x-u\| .
\end{aligned}
$$

This shows $\left(x_{n}\right)$ is bounded and so is $\left(y_{n}\right)$.

STEP 2. $\lim _{n \rightarrow \infty}\left\|y_{n}-x_{n}\right\|=0$.

It follows from (3.1) that

$$
\left\|y_{n}-y_{n-1}\right\|=\left\|J_{M, \lambda}(I-\lambda A) x_{n}-J_{M, \lambda}(I-\lambda A) x_{n-1}\right\| \leq\left\|x_{n}-x_{n-1}\right\|,
$$

and also that

$$
\begin{aligned}
\left\|x_{n+1}-x_{n}\right\| & \leq\left\|\left[\alpha_{n} x+\left(1-\alpha_{n}\right) y_{n}\right]-\left[\alpha_{n-1} x+\left(1-\alpha_{n-1}\right) y_{n-1}\right]\right\| \\
& =\left\|\left(\alpha_{n}-\alpha_{n-1}\right)\left(x-y_{n-1}\right)+\left(1-\alpha_{n}\right)\left(y_{n}-y_{n-1}\right)\right\| \\
& \leq\left|\alpha_{n}-\alpha_{n-1}\right|\left\|x-y_{n-1}\right\|+\left(1-\alpha_{n}\right)\left\|x_{n}-x_{n-1}\right\| \\
& \leq M\left|\alpha_{n}-\alpha_{n-1}\right|+\left(1-\alpha_{n}\right)\left\|x_{n}-x_{n-1}\right\|,
\end{aligned}
$$

where $M=\left(\|x\|+\sup _{n \geq 0}\left\|y_{n}\right\|\right)$. By virtue of conditions (C1) and (C2), we can apply Lemma 2.4 to 3.2 to obtain $x_{n+1}-x_{n} \rightarrow 0$. Consequently, we also have

$$
\lim _{n \rightarrow \infty}\left\|x_{n}-y_{n}\right\|=0
$$


In fact, since $J_{M, \lambda}(I-\lambda A)$ is averaged, we may assume that it is $\kappa$-averaged for some $\kappa \in(0,1)$. Then it follows from Lemma 2.1 (v) that

$$
\begin{aligned}
\left\|x_{n+1}-u\right\|^{2} & \leq\left\|\alpha_{n} x+\left(1-\alpha_{n}\right) y_{n}-u\right\|^{2} \\
& \leq \alpha_{n}\|x-u\|^{2}+\left(1-\alpha_{n}\right)\left\|y_{n}-u\right\|^{2} \\
& \leq \alpha_{n}\|x-u\|^{2}+\left\|J_{M, \lambda}(I-\lambda A) x_{n}-u\right\|^{2} \\
& \leq \alpha_{n}\|x-u\|^{2}+\left\|x_{n}-u\right\|^{2}-\frac{1-\kappa}{\kappa}\left\|J_{M, \lambda}(I-\lambda A) x_{n}-x_{n}\right\|^{2} \\
& =\alpha_{n}\|x-u\|^{2}+\left\|x_{n}-u\right\|^{2}-\frac{1-\kappa}{\kappa}\left\|y_{n}-x_{n}\right\|^{2} .
\end{aligned}
$$

Letting $L=2 \sup _{n \geq 0}\left\|x_{n}\right\|$, we get

$$
\begin{aligned}
\frac{1-\kappa}{\kappa}\left\|y_{n}-x_{n}\right\|^{2} & \leq\left\|x_{n}-u\right\|^{2}-\left\|x_{n+1}-u\right\|^{2}+\alpha_{n}\|x-u\|^{2} \\
& \leq L\left\|x_{n}-x_{n+1}\right\|+\alpha_{n},
\end{aligned}
$$

and therefore (3.3) follows from (3.4) by tending $n \rightarrow \infty$.

STEP 3. If $z \in \omega_{w}\left(x_{n}\right)$, then $z \in F(S) \cap V I(\mathcal{H}, A, M)$.

To see this, we set $z_{n}=\alpha_{n} x+\left(1-\alpha_{n}\right) y_{n}$. Then we conclude that

$$
\left\|x_{n}-z_{n}\right\| \leq\left\|x_{n}-y_{n}\right\|+\left\|y_{n}-z_{n}\right\|=\left\|x_{n}-y_{n}\right\|+\alpha_{n}\left\|x-y_{n}\right\| \rightarrow 0, \text { as } n \rightarrow \infty,
$$

which further gives that

$$
\left\|S z_{n}-z_{n}\right\| \leq\left\|x_{n+1}-x_{n}\right\|+\left\|x_{n}-z_{n}\right\| \rightarrow 0, \text { as } n \rightarrow \infty .
$$

Take a subsequence $\left(x_{n_{k}}\right)$ of $\left(x_{n}\right)$ such that $x_{n_{k}} \rightarrow z$; hence $x_{n_{k}} \rightarrow z$. By Lemma 2.3, we have $z \in F(S)$. Since

$$
\left\|J_{M, \lambda}(I-\lambda A) x_{n}-x_{n}\right\|=\left\|y_{n}-x_{n}\right\| \rightarrow 0, \text { as } n \rightarrow \infty,
$$

we get, by using Lemma 2.3 again, $z \in F\left(J_{M, \lambda} V_{\beta}\right)=V I(\mathcal{H}, A, M)$.

STEP 4. $x_{n} \rightarrow x^{*}:=P_{F(S) \cap V I(\mathcal{H}, A, M)} x$.

It follows from the definition of $x^{*}$ that

$$
\begin{aligned}
\left\|x_{n+1}-x^{*}\right\|^{2} & \leq\left\|\alpha_{n} x+\left(1-\alpha_{n}\right) y_{n}-x^{*}\right\|^{2} \\
& =\left(1-\alpha_{n}\right)^{2}\left\|y_{n}-x^{*}\right\|^{2}+\alpha_{n}^{2}\left\|x-x^{*}\right\|^{2}+2 \alpha_{n}\left(1-\alpha_{n}\right)\left\langle y_{n}-x^{*}, x-x^{*}\right\rangle \\
& \leq\left(1-\alpha_{n}\right)\left\|x_{n}-x^{*}\right\|^{2}+\alpha_{n}^{2}\left\|x-x^{*}\right\|^{2}+2 \alpha_{n}\left(1-\alpha_{n}\right)\left\langle y_{n}-x^{*}, x-x^{*}\right\rangle \\
& =\left(1-\alpha_{n}\right)\left\|x_{n}-x^{*}\right\|^{2}+\alpha_{n}^{2}\left\|x-x^{*}\right\|^{2}+2 \alpha_{n}\left(1-\alpha_{n}\right)\left\langle x_{n}-x^{*}, x-x^{*}\right\rangle .
\end{aligned}
$$

In view of Lemma 2.4, if we show that

$$
\varlimsup_{n \rightarrow \infty}\left\langle x_{n}-x^{*}, x-x^{*}\right\rangle \leq 0,
$$

then the proof is finished. To this end, let $\left(x_{n_{k}}\right)$ be a subsequence of $\left(x_{n}\right)$ converging weakly to $z$ and

$$
\varlimsup_{n \rightarrow \infty}\left\langle x_{n}-x^{*}, x-x^{*}\right\rangle=\lim _{k \rightarrow \infty}\left\langle x_{n_{k}}-x^{*}, x-x^{*}\right\rangle .
$$

By Step 3, $z \in F(S) \cap V I(\mathcal{H}, A, M)$. This together with 2.1 and $x^{*}:=P_{F(S) \cap V I(\mathcal{H}, A, M)} x$ implies that

$$
\varlimsup_{n \rightarrow \infty}\left\langle x_{n}-x^{*}, x-x^{*}\right\rangle=\left\langle z-x^{*}, x-x^{*}\right\rangle \leq 0,
$$

which is the result as desired. 
Analogously, by using the viscosity approximation method, one can easily get some other algorithms for approximating a solution to problem (1.5).

Theorem 3.6. Let $A: \mathcal{H} \rightarrow \mathcal{H}$ be $\alpha$-ism with $\alpha>0, M: \mathcal{H} \rightarrow 2^{\mathcal{H}}$ a maximal monotone mapping, $f: \mathcal{H} \rightarrow \mathcal{H}$ a contractive mapping, and $S: \mathcal{H} \rightarrow \mathcal{H}$ a nonexpansive mapping. Choose $\lambda \in(0,2 \alpha)$ and define a sequence $\left(x_{n}\right)$ by the iterative procedure:

$$
\left\{\begin{array}{l}
x_{0}=x \in \mathcal{H} \\
y_{n}=J_{M, \lambda}\left(x_{n}-\lambda A x_{n}\right) \\
x_{n+1}=\alpha_{n} f\left(x_{n}\right)+\left(1-\alpha_{n}\right) S y_{n}
\end{array}\right.
$$

If $\left(\alpha_{n}\right)$ is chosen in $(0,1)$ so that conditions $(\mathrm{C} 1)$ and $(\mathrm{C} 2)$ are satisfied, then the sequence $\left(x_{n}\right)$ generated by 3.5 converges strongly to $x^{*}=P_{F(S) \cap V I(\mathcal{H}, A, M)} f$, whenever $F(S) \cap V I(\mathcal{H}, A, M) \neq \emptyset$.

Theorem 3.7. Let $A: \mathcal{H} \rightarrow \mathcal{H}$ be $\alpha$-ism with $\alpha>0, M: \mathcal{H} \rightarrow 2^{\mathcal{H}}$ a maximal monotone mapping, $f: \mathcal{H} \rightarrow \mathcal{H}$ a contractive mapping, and $S: \mathcal{H} \rightarrow \mathcal{H}$ a nonexpansive mapping. Choose $\lambda \in(0,2 \alpha)$ and define a sequence $\left(x_{n}\right)$ by the iterative procedure:

$$
\left\{\begin{array}{l}
x_{0}=x \in \mathcal{H} \\
y_{n}=J_{M, \lambda}\left(x_{n}-\lambda A x_{n}\right), \\
x_{n+1}=S\left[\alpha_{n} f\left(x_{n}\right)+\left(1-\alpha_{n}\right) y_{n}\right]
\end{array}\right.
$$

If $\left(\alpha_{n}\right)$ is chosen in $(0,1)$ so that the conditions $(\mathrm{C} 1)$ and $(\mathrm{C} 2)$ are satisfied, then the sequence $\left(x_{n}\right)$ generated by (3.6) converges strongly to $x^{*}=P_{F(S) \cap V I(\mathcal{H}, A, M)} f$, whenever $F(S) \cap V I(\mathcal{H}, A, M) \neq \emptyset$.

\section{References}

[1] C. Byrne, A unified treatment of some iterative algorithms in signal processing and image reconstruction, Inverse Problems, 20 (2004), 103-120. 2.1, 3.2

[2] B. Halpern, Fixed points of nonexpanding maps, Bull. Amer. Math. Soc., 73 (1967), 957-961. 2

[3] H. Iiduka, W. Takahashi, Strong convergence theorems for nonexpansive mappings and inverse-strongly monotone mappings, Nonlinear Anal., 61 (2005), 341-350. 1

[4] A. Moudafi, Viscosity approximation methods for fixed-points problems, J. Math. Anal. Appl., 241 (2000), 46-55. 2

[5] N. Nadezhkina, W. Takahashi, Weak convergence theorem by an extragradient method for nonexpansive mappings and monotone mappings, J. Optim. Theory Appl., 128 (2006), 191-201. 1

[6] W. Takahashi, M. Toyoda, Weak convergence theorems for nonexpansive mappings and monotone mappings, J. Optim. Theory Appl., 118 (2003), 417-428. 1, 1, 1

[7] R. Wittmann, Approximation of fixed points of nonexpansive mappings, Arch. Math. (Basel), 58 (1992), $486-491$. 2

[8] H.-K. Xu, Iterative algorithms for nonlinear operators, J. London Math. Soc. (2), 66 (2002), 240-256. 2, 2.4

[9] H.-K. Xu, Viscosity approximation methods for nonexpansive mappings, J. Math. Anal. Appl., 298 (2004), 279291. 2

[10] L.-C. Zeng, J.-C. Yao, Strong convergence theorem by an extragradient method for fixed point problems and variational inequality problems, Taiwanese J. Math., 10 (2006), 1293-1303. 1

[11] S.-S. Zhang, J. H. W. Lee, C. K. Chan, Algorithms of common solutions to quasi variational inclusion and fixed point problems, Appl. Math. Mech. (English Ed.), 29 (2008), 571-581. 1, 1, $2,3,3.4$ 